\title{
Explorer l'architecture du vivant à l'échelle moléculaire grâce à la microscopie de super-résolution PALM/STORM
}

\author{
Ignacio IZEDDIN, Xavier DARZACQ, \\ Functional Imaging of Transcription, CNRS UMR8197, École Normale Supérieure, 75005 Paris \\ Maxime DAHAN \\ Institut de Biologie de l'ENS, IBENS, 75005 Paris \\ Laboratoire Physico-Chimie, Institut Curie et CNRS UMR 168, 75005 Paris \\ maxime.dahan@curie.fr
}

L'étude de nombreux mécanismes en biologie requiert de décrire de manière quantitative l'organisation spatiale et la dynamique de systèmes vivants. Par sa spécificité moléculaire et sa compatibilité avec le vivant, la microscopie de fluorescence est depuis longtemps un outil de référence en biologie. Cependant, un inconvénient de la microscopie optique est que sa résolution spatiale est limitée par la nature ondulatoire de la lumière, à typiquement $250 \mathrm{~nm}$ dans le plan $x-y$ et $500 \mathrm{~nm}$ selon l'axe optique $z$ (figure 1a). C'est donc un outil très puissant pour analyser la morphologie des cellules (dont la taille typique est comprise entre 1 et $100 \mathrm{~mm}$ ) mais bien moins approprié pour étudier les propriétés d'assemblages macromoléculaires (à l'échelle 10-100 nm) dont on sait pourtant qu'ils sont essentiels pour le fonctionnement de nos cellules.

\section{Une limite de résolution de l'ordre de $250 \mathrm{~nm}$}

La limite de résolution n'est autre que la conséquence du phénomène de diffraction de la lumière à travers un système optique. Comme l'a décrit Ernst Abbe dès 1873 , l'image d'un point source de lumière, c'est-à-dire la réponse impulsionnelle d'un microscope, est une tache de diffraction (souvent appelée PSF, pour point spread function en anglais). Le profil d'intensité de cette tache de diffraction suit la forme théorique d'un disque d'Airy, souvent approximé par une courbe Gaussienne. La résolution planaire d'une image peut donc être définie comme la distance minimale à laquelle on peut distinguer les taches de diffraction de deux points sources de lumière. Selon le critère d'Abbe, la largeur à mi-hauteur de la tache de diffraction définit cette distance, la résolution d'une image est ainsi déterminée par $\lambda / 2 O N$ où $\lambda$ est la longueur d'onde de la lumière observée, et $O N$ l'ouverture numérique du système optique. Selon le critère de Rayleigh, plus généralisé que celui d'Abbe, la puissance résolutive d'un système optique se définit comme la distance $d_{\text {Ray }}$ entre le centre de la fonction d'Airy et le premier minimum de

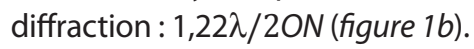

Indépendamment du critère choisi, il est important de noter que les seuls paramètres qui influencent la résolution d'une image microscopique sont la longueur d'onde et l'ouverture numérique du microscope. Typiquement, en biologie, où l'on travaille avec de la lumière visible (450-700 nm) et des objectifs dont l'ON peut aller jusqu'à 1,4 , la résolution de l'image est d'environ $250 \mathrm{~nm}$.

\section{De la détection de la molécule unique au principe de super-résolution PALM/STORM}

À cause de sa nature physique fondamentale, la limite de diffraction a longtemps été considérée comme une barrière infranchissable en imagerie optique.
Seule la microscopie électronique, pour laquelle la longueur d'onde est plus petite que celle des photons visibles de plus de cinq ordres de grandeurs, permettait d'accéder aux informations structurales à l'échelle moléculaire. Pourtant, au cours des dix dernières années, deux stratégies conceptuellement différentes ont montré qu'il était en fait possible de contourner la limite de résolution imposée par la diffraction de la lumière, ouvrant ainsi la voie à une véritable révolution pour l'imagerie biologique : la microscopie super-résolutive ou nanoscopie.

La première méthode, que nous ne détaillerons pas dans cet article, consiste à manipuler le profil spatial des faisceaux d'illumination. La microscopie STED (stimulated emission depletion) et la microscopie d'illumination structurée SIM (structured illumination microscopy) sont deux exemples importants. La deuxième stratégie, présentée ici, est basée sur l'imagerie de molécules uniques et a donné naissance aux microscopies dites PALM (photoactivated localization microscopy) 
et STORM (stochastic optical reconstruction microscopy).

\section{Détecter des molécules uniques grâce aux sondes fluorescentes photoactivables}

Les techniques PALM/STORM ont été rendues possibles par la combinaison de deux éléments : des sondes fluorescentes photoactivables et la détection optique de molécules individuelles. En effet, il existe depuis quelques années des sondes photoactivables, c'est-à-dire dont on peut contrôler l'activité de fluorescence dans le visible à l'aide d'un signal optique de plus grande longueur d'onde. En d'autres termes, lorsqu'on les illumine avec de la lumière violette (le plus souvent à $405 \mathrm{~nm}$ ), ces molécules transitent d'un état inactif dans lequel elles n'émettent pas de lumière, vers un état actif dans lequel elles peuvent émettre des photons de fluorescence visibles. Ainsi, en réduisant l'intensité de la lumière violette, on réduit le nombre de molécules actives, jusqu'à un régime où il n'y a que quelques molécules dont la distance relative est supérieure à la limite de diffraction. Chaque molécule agissant comme une source ponctuelle, son image est donc donnée par une tache de diffraction (la PSF). Lorsqu'on sait qu'il s'agit du signal d'une seule molécule, il est possible de déterminer sa position avec une précision bien meilleure que l'étalement de cette tache. En modélisant par exemple la PSF expérimentale avec une fonction gaussienne, on peut ainsi déterminer par un ajustement de l'image les coordonnés $\left(x_{0}, y_{0}\right)$ de l'émetteur avec une précision proportionnelle à la longueur d'onde divisée par la racine carrée du nombre de photons détectés (figure 1c). On comprend alors aisément comment obtenir des images avec une résolution sous la limite de diffraction. Au lieu d'utiliser des sondes qui émettent de la lumière simultanément, on marque les objets d'intérêt avec des sondes photoactivables. On répète alors la séquence suivante: on active quelques molécules, on les détecte individuellement avec une grande précision, on les éteint par photoblanchiement. Ainsi, en séparant temporellement la détection des
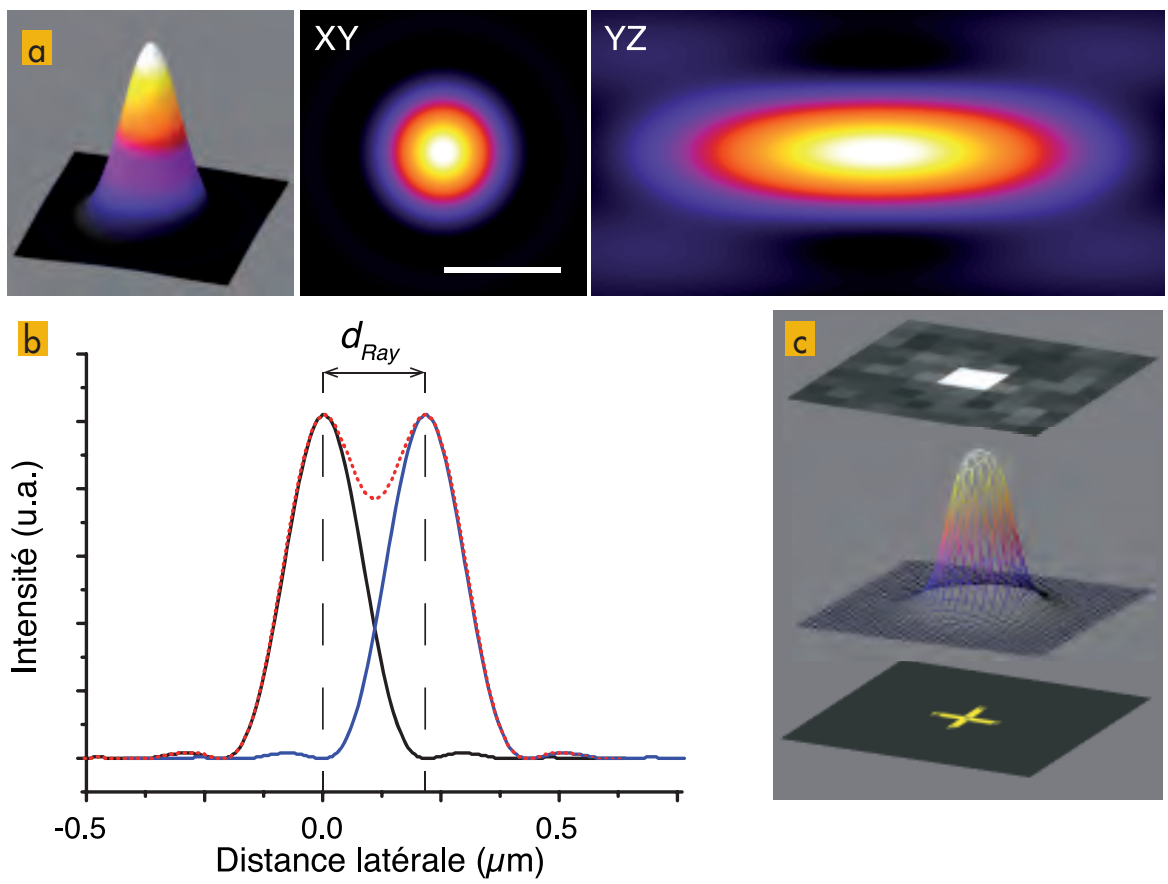

Figure 1. Diffraction de la lumière et limite de résolution. La réponse impulsionnelle d'un microscope d'un point source de lumière est une tâche de diffraction d'une taille de typiquement $250 \mathrm{~nm}$ en xy et $500 \mathrm{~nm}$ en $z$, comme montré dans la partie (a) de la figure, où on représente une PSF (pointspread function) simulée avec une $O N=1,4$ et $\lambda=500 \mathrm{~nm}$. La barre d'échelle correspond à la distance de Rayleigh $d_{\text {Ray }}=260 \mathrm{~nm}$. (b) Profils d'intensité de deux fonctions d'Airy à une distance considérée comme la limite de résolution par le critère de Rayleigh. (c) Principe de pointage d'une image molécule unique par ajustement gaussien.
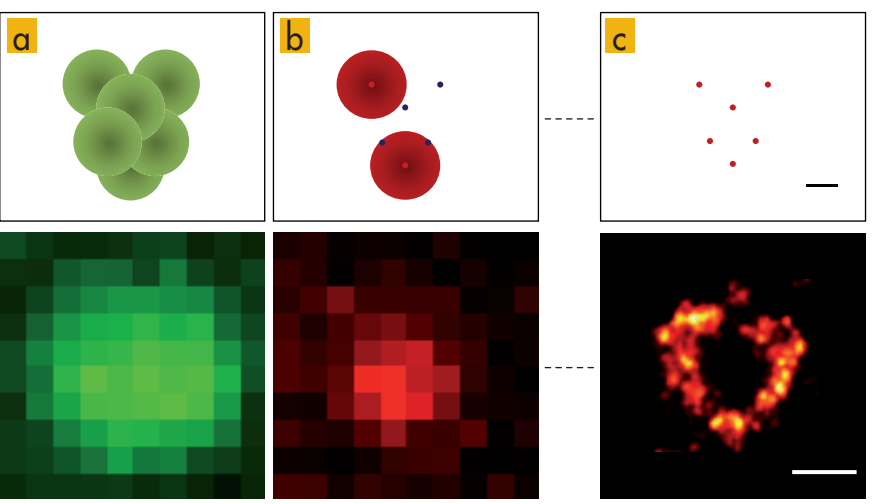

molécules composant l'échantillon, on en reconstruit l'image totale avec une résolution donnée par la précision de localisation et non plus par la limite de diffraction (figure 2). Un point important est que le nombre de points doit être suffisamment élevé pour satisfaire le critère d'échantillonnage de NyquistShannon qui stipule que la densité de localisation doit être au moins égaleà ( $2 /$ résolution) ${ }^{\mathrm{D}}$ (où

Figure 2. Principe de super-résolution par détection de molécule unique. (a) La limite de résolution en microscopie conventionnelle résulte de l'impossibilité de distinguer des objets proches émetteurs de lumière. (b) En séparant temporellement l'émission des fluorophores marquant une structure, ils peuvent être détectés comme des émetteurs uniques et leurs positions déterminées avec une résolution nanométrique. (c) L'image super-résolve peut être reconstruite à partir des localisations de toutes les molécules détectées. Les images inférieures proviennent d'une expérience PALM où la protéine Cep 123, qui fait partie du complexe du centrosome, a été marquée avec la protéine photo-convertible mEos2 [4] (barre d'échelle, $250 \mathrm{~nm}$ ).
Dest la dimension de l'objet à reconstruire).

\section{Bien choisir la sonde photoactivable}

Les méthodes de microscopie de super-résolution par détection de molécules individuelles photoactivables ont été développées par trois équipes américaines en 2006 (voir revue dans [1]). Leur principe est le même mais ces méthodes diffèrent par le type des sondes utilisées: des protéines fluorescentes pour la microscopie PALM et des colorants organiques pour le STORM. Chacune des approches a ses avantages et inconvénients respectifs. En particulier, le 
rendement de fluorescence des colorants organiques est souvent plus élevé que celui des protéines fluorescentes, ce qui permet d'obtenir des précisions de pointé bien meilleures. Toutefois, les protéines photoactivables peuvent être encodées génétiquement, ce qui est souvent une meilleure stratégie de marquage et facilite l'imagerie dans les cellules vivantes.

En général, la résolution de la microscopie PALM/STORM peut atteindre des valeurs de 20 à $30 \mathrm{~nm}$, soit environ un ordre de grandeur inférieur à la limite de diffraction. Ce gain, associé à la simplicité technique du montage expérimental, a fait du PALM/STORM une technique de microscopie rapidement adoptée par un grand nombre de laboratoires, ce qui a déjà permis l'étude d'une multitude de structures subcellulaires avec une résolution sans précédent.

\section{Imagerie de super-résolution tridimensionnelle et multicouleur}

\section{Vers des images super-résolues tridimentionnelles}

Nous avons montré ci-dessus comment la microscopie PALM/STORM permettait d'améliorer la résolution des images bidimensionnelles. Toutefois, la plupart des systèmes biologiques ne sont pas plans et obtenir des images de microscopie super-résolues tridimensionnelles est un enjeu essentiel. S'il est relativement aisé de déterminer la position d'une molécule individuelle dans le plan image $x$ - $y$ avec une précision de l'ordre de $20 \mathrm{~nm}$, le faire selon l'axe optique $z$ est plus délicat. En effet, dans la profondeur du champ du microscope (environ 0,5 à $1 \mu \mathrm{m}$ en pratique), l'image d'une molécule individuelle est une tache de diffraction plus ou moins défocalisée selon la position de la source relative au plan image. Malheureusement, cette image varie peu au voisinage du plan focal et, de plus, elle possède une symétrie par rapport à ce plan, ce qui empêche de déterminer si la molécule se trouve au-dessus ou en dessous du plan focal.

II existe plusieurs solutions pour surmonter ces difficultés et nous présentons ici les deux plus courantes. Une première méthode consiste à réaliser une ingénierie de la PSF de manière à encoder précisément la localisation 3D dans l'image. En plaçant une lentille cylindrique dans le chemin optique d'émission, on induit par exemple un astigmatisme qui brise la symétrie axiale de la PSF. L'image d'une molécule unique est maintenant un ellipsoïde dont la largeur selon les axes $x$ et $y$ est fonction de la distance au plan focal (figure 3a). Une version plus élaborée consiste à remplacer la lentille cylindrique par un miroir déformable et à appliquer les méthodes d'optique adaptative pour modifier avec soin le front d'onde de la lumière émise [2]. Une deuxième approche consiste à imager la molécule simultanément dans deux plans légèrement séparés (de l'ordre de $400 \mathrm{~nm}$ ), ce qui permet d'en inférer la position en $z$ (figure $3 b$ ). La précision de localisation selon $z$ des méthodes astigmatique et bi-plan est similaire, approximativement deux fois la précision en $x-y$. Toutefois, une limitation actuelle des méthodes de localisation 3D est qu'elles ne fonctionnent que sur des épaisseurs ne dépassant pas un ou deux microns.

\section{Observer plusieurs espèces moléculaires}

Parallèlement aux efforts pour étendre la super-résolution aux trois dimensions de l'espace, il y a un besoin d'observer dans une même image plusieurs espèces moléculaires. Pour cela, il est nécessaire d'utiliser des sondes photoactivables ayant des longueurs d'onde d'émission distinctes. Aujourd'hui, en combinant des outils de PALM et STORM, on sait produire des images multicouleurs dans lesquelles 2 ou 3 protéines sont spécifiquement marquées (figure 3c). On peut alors en analyser les localisations relatives des protéines et tester ainsi leurs interactions potentielles.

\section{Dynamique structurelle et moléculaire}

Les images PALM/STORM sont reconstituées à partir de centaines de milliers ou de millions de détections de molécules individuelles. Pour obtenir un aussi grand nombre de détections, il est nécessaire d'acquérir de longues séquences, pouvant atteindre $10^{5}$ images. Ceci a longtemps limité les études à des échantillons fixés, ne

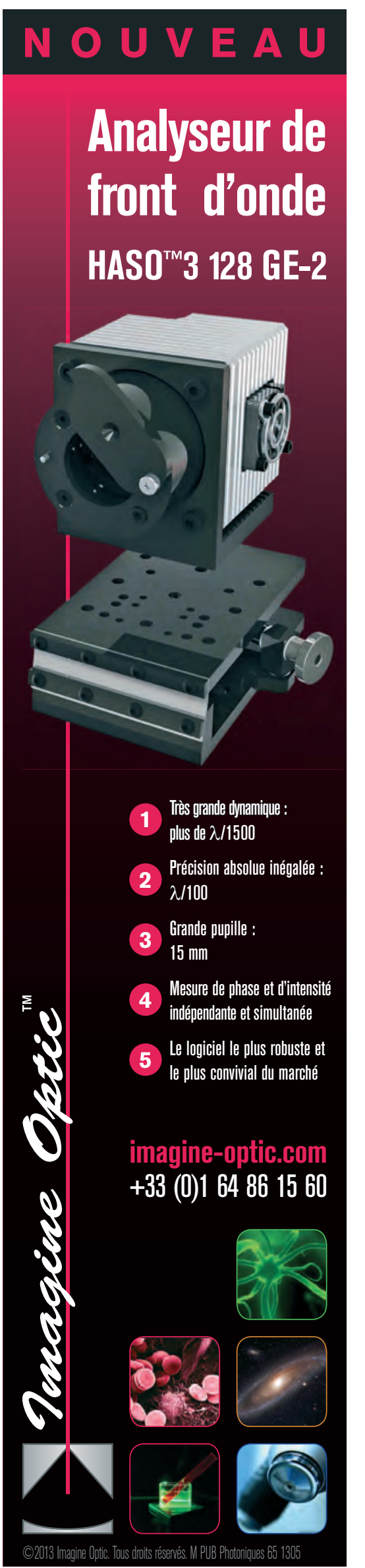




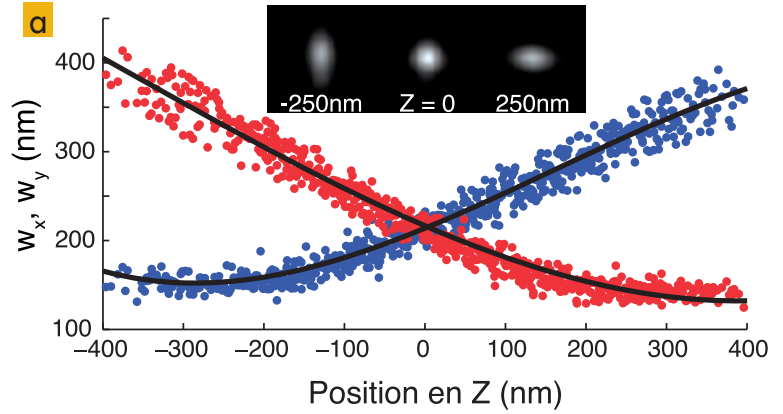

b

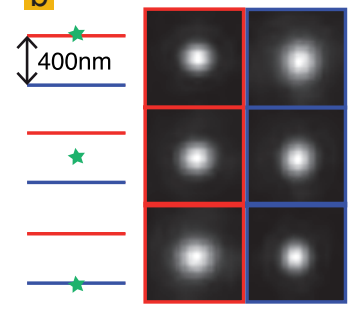

c

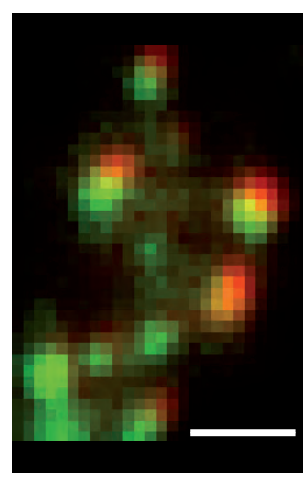

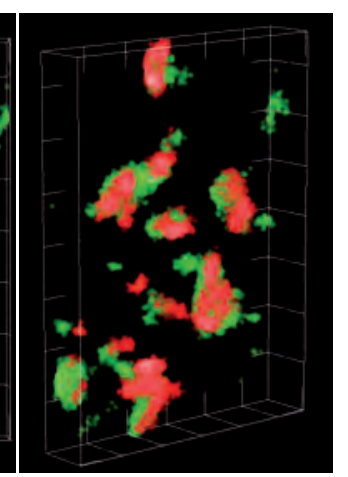

Figure 3. Microscopie PALM / STORM en 3D et en deux couleurs. (a) Lorsqu'on place une lentille cylindrique sur le chemin d'émission, la forme de la PSF est déformée en $x$ et en $y$ en fonction de la distance au plan focal. Après calibration des largueurs $w_{x}$ et $w_{y^{\prime}}$ on peut déterminer avec une haute précision la position en $z$ des molécules détectées. (b) D'une façon similaire, la détection d'une même molécule avec deux caméras qui imagent deux plans focaux légèrement décalés, permet de déterminer la position axiale de ces molécules. (c) Exemple d'image PALM/STORM en deux couleurs et trois dimensions en utilisant I'approche astigmatique. L'image montre la co-localisation de la protéine géphyrine (en vert) et les récepteurs de glycine GlyRal (en rouge) dans des cellules neuronales de la moelle épinière [5]. A gauche, l'image acquise en microscopie de champ large conventionnelle (barre d'échelle, $1 \mu \mathrm{m}$ ) ; au centre, l'image super-résolue en vision frontale $(x y)$; à droite, une représentation tridimensionnelle de la même image.

permettant pas d'aborder la dynamique fonctionnelle des structures biologiques. Dans les dernières années, la microscopie de super-résolution a été étendue à des images dynamiques, avec une résolution temporelle de l'ordre de la seconde en STORM et de l'ordre de vingt secondes en PALM. Le gain en résolution temporelle n'est toutefois pas toujours sans effet sur la résolution spatiale. En effet, lorsqu'on réduit la durée d'acquisition, et donc le nombre de molécules utilisées pour reconstruire l'image, le facteur limitant la résolution spatiale peut ne plus être la précision de localisation des molécules individuelles, mais la densité inférieure de détections.

Le développement des protéines photoactivables permet non seulement de reconstituer l'image de structures cellulaires, mais aussi d'étudier les mécanismes qui contrôlent la mobilité à l'échelle moléculaire (figure 4). Pour cela, on active des molécules, toujours de manière peu dense, puis on en suit le mouvement jusqu'à photodestruction du fluorophore [3]. Ces trajectoires individuelles permettent de déterminer les processus de transport, diffusif ou dirigé, mais aussi les cinétiques d'interaction avec des partenaires moléculaires. Les approches par photoactivation offrent deux avantages importants par rapport aux techniques habituelles de suivi de molécules dans
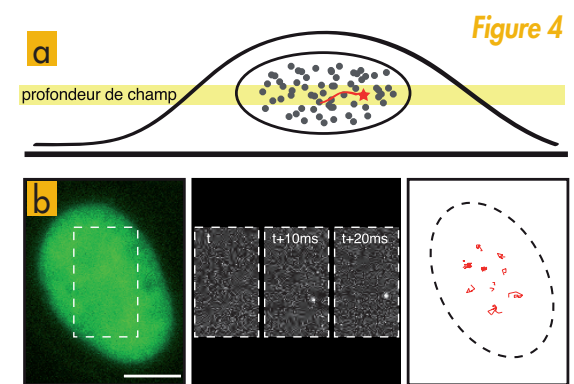

lesquelles on fait un marquage des protéines d'intérêt via des sondes organiques ou des nanoparticules. Tout d'abord, elles permettent d'étudier des protéines dans des compartiments cellulaires, tels que le noyau, difficiles d'accès à des marqueurs exogènes. De plus, comme on peut répéter séquentiellement les phases d'activation et d'acquisition, on a accès à un grand nombre de trajectoires. On peut donc mettre en place des techniques computationnelles puissantes pour cartographier la cellule et mieux décrire l'hétérogénéité du milieu cellulaire.

En conclusion, les nouvelles méthodes de microscopie optique par photoactivation donnent accès à des informations structurales et dynamiques dans la cellule, avec une résolution spatiale sans précédent. Elles contribuent ainsi à lever le voile sur le lien complexe entre l'organisation spatiale des molécules et les fonctions cellulaires.

\section{Remerciements}

Nous remercions James Sillibourne, Michel Bornens, Mohamed El-Beheiry, Vincent Récamier, Christian Specht et Antoine Triller pour leur participation à l'acquisition et l'analyse des données présentées dans les figures.

\section{Références}

[1] Huang B., Babcock H., Zhuang X., Breaking the diffraction barrier: super-resolution imaging of cells. Cell 143(7), 1047-1058 (2010).

[2] Izeddin I., El Beheiry M., Andilla J., Ciepielewski D., Darzacq X., Dahan M., PSF shaping using adaptive optics for three-dimensional single-molecule super-resolution imaging and tracking. Opt Express 20(5), 4957-4967 (2012).

[3] Manley S., Gillette J.M., Patterson G.H., Shroff H., Hess H.F., Betzig E., Lippincott-Schwartz J., High-density mapping of single-molecule trajectories with photoactivated localization microscopy. Nat Methods 5, 155-157 (2008).

[4] Sillibourne J.E., Specht C.G., Izeddin I., Hurbain I., Tran P., Triller A., Darzacq X., Dahan M., Bornens M., Assessing the localization of centrosomal proteins by PALM/STORM nanoscopy. Cytoskeleton 68(1 1), 619-627 (2011)

[5] Specht C.G., Izeddin I., Rodriguez P.C., El Beheiry M., Rostaing P., Darzacq X., Dahan M., Triller A., Quantitative nanoscopy of inhibitory synapses: counting gephyrin molecules and receptor binding sites. Neuron, in press (2013) 